\title{
Tregs in T cell vaccination: exploring the regulation of regulation
}

\author{
Irun R. Cohen, Francisco J. Quintana, and Avishai Mimran
}

Department of Immunology, The Weizmann Institute of Science, Rehovot, Israel.

\begin{abstract}
T cell vaccination (TCV) activates Tregs of 2 kinds: anti-idiotypic (anti-id) and anti-ergotypic (anti-erg). These regulators furnish a useful view of the physiology of $T$ cell regulation of the immune response. Anti-id Tregs recognize specific effector clones by their unique TCR CDR3 peptides; anti-id networks of $\mathrm{CD}^{+}{ }^{+}$and $\mathrm{CD8}^{+} \mathrm{Tregs}$ have been described in detail. Here we shall focus on anti-erg T regulators. Anti-erg T cells, unlike anti-id T cells, do not recognize the clonal identity of effector $T$ cells; rather, anti-erg $T$ cells recognize the state of activation of target effector $T$ cells, irrespective of their TCR specificity. We consider several features of anti-erg T cells: their ontogeny, subset markers, and target ergotope molecules; mechanisms by which they regulate other $T$ cells; mechanisms by which they get regulated; and therapeutic prospects for anti-erg upregulation and downregulation.
\end{abstract}

\begin{abstract}
History
T cell vaccination (TCV) became possible once we discovered how to raise and maintain in vitro autoimmune $T$ cell lines and clones capable of adoptively transferring experimental autoimmune encephalomyelitis (EAE) to naive recipients (1). If EAE could be reduced to the actions of defined anti-myelin $\mathrm{T}$ cells, might EAE be resisted or aborted by vaccinating test rats with these same anti-myelin $\mathrm{T}$ cells suitably attenuated? The answer turned out to be yes; irradiated $\mathrm{T}$ cells specific for myelin basic protein (MBP) could indeed, when used as a vaccine, protect against future EAE induced by active autoimmunization to MBP (2). Surprisingly, vaccination with as few as one thousand $T$ cells of a single clone could lead to the downregulation of as complex a disease as adjuvant arthritis (3). We concluded that the immune system could respond with great sensitivity to vaccination with its own agents and that the mechanism of immune regulation induced by TCV with a single clone could actively modify an entire polyclonal response (3). TCV was applied to various autoimmune disease models, including spontaneous diseases such as murine lupus (4) and type 1 diabetes mellitus (5), and the procedure is now being applied to human disease, mostly multiple sclerosis, by the groups of Zhang (6), Raus (7), Weiner (8), and others (9). But the remarkable sensitivity and effectiveness of TCV supported the idea that TCV might afford a view of natural immune regulation (10).
\end{abstract}

\section{Antigen-specific anti-id networks}

The first regulators noted to be induced by TCV were anti-idiotypic (anti-id) T cells; both $\mathrm{CD}^{+}$and $\mathrm{CD}^{+}$anti-id T cells were detected in mice following TCV against EAE (11). TCR peptides were shown to be targets for regulation (5), demonstrating unequivocally that TCV could induce anti-id immunity. Recent work by Jiang, Chess, and colleagues (12), Kumar, Sercarz, and colleagues (13), and Cantor and colleagues $(14,15)$ is revealing the complexity of the antiid network of Tregs. A chain reaction begins with $\mathrm{CD}^{+}$anti-id $\mathrm{T}$

Nonstandard abbreviations used: anti-erg, anti-ergotypic; anti-id, anti-idiotypic; EAE, experimental autoimmune encephalomyelitis; IL-2R, IL-2 receptor; MBP, myelin basic protein; TCV, $\mathrm{T}$ cell vaccination.

Conflict of interest: The authors have declared that no conflict of interest exists.

Citation for this article: J. Clin. Invest. 114:1227-1232 (2004).

doi:10.1172/JCI200422396. cells that respond to TCR peptides presented by the autoimmune effector $\mathrm{T}$ cells. These anti-id CD4 ${ }^{+} \mathrm{T}$ cells, in turn, activate $\mathrm{CD} 8^{+}$ $\mathrm{T}$ cells that actually downregulate the autoimmune effector $\mathrm{T}$ cells responsible for the autoimmune disease. The $\mathrm{CD} 8^{+}$Tregs appear to recognize their target TCR peptides presented by Qa-1/HLA-E MHC class Ib molecules $(12,14,15)$.

True anti-id $\mathrm{T}$ regulators would, by definition, recognize a peptide from the domain of the TCR that is unique to the clone that expresses that TCR; such clonally unique peptides would have to include the CDR3 region of the TCR (5). However, peptides from non-clonally restricted domains of the TCR, both within the TCR $\mathrm{V}$ segment (16) and in framework regions of the TCR (17), have also been found to be targeted by Tregs. Thus, not all TCR-directed Tregs are anti-ids. $\mathrm{T}$ cell-directed regulation that is not idiotypic brings us to the subject of anti-ergotypic (anti-erg) regulation.

\section{Anti-erg T cells}

In order for lines and clones of autoimmune effector $\mathrm{T}$ cells to adoptively transfer EAE or other experimental diseases, the effector cells must be activated in vitro before transfer; one million activated anti-MBP T cells could cause EAE, but fifty million of the same $T$ cells in a resting, post-activation state could not cause EAE (2). The need for activation to cause EAE was fairly easy to understand; only activated effector T cells migrated through blood vessels and entered the central nervous system (18). Chemokine receptors, adhesion molecules, and other factors needed for effector $\mathrm{T}$ cell homing do require $\mathrm{T}$ cell activation (19). More surprising was the observation that activated $\mathrm{T}$ cells were required to make a vaccine for $\mathrm{TCV}$; resting $\mathrm{T}$ cells were unable to provide protection (10). If the TCR was the target of the T regulators, why did only activated T cells vaccinate? Resting T cells express at least as much TCR as do activated T cells. So what can T cell activation add to an anti-id immune response? One could imagine that activation might be needed to induce in the $\mathrm{T}$ cell vaccine some additional but then-unknown adjuvant molecules.

The requirement for activation was a nagging problem, and its resolution led to experiments with TCV against EAE using activated T cells whose TCR was not specific for MBP, the target antigen in rat EAE. Unexpectedly, TCV with activated T cells of other antigen specificities seemed to be able to vaccinate rats significantly against $\mathrm{EAE}(20)$. The vaccination was specific for the activation 
of the vaccine $T$ cells (only activated $T$ cells worked) but was not specific for the TCR identity of the vaccine (some stock-activated $\mathrm{T}$ cell lines could downregulate EAE, at least to some extent).

The observation of effective TCV with TCR nonspecificity was disconcerting, but we were able at least to coin a term: even without knowing their identity, we named the necessary activation molecules "ergotopes" (from the Greek ergon, meaning "work" or "activity"). Thus, the regulators induced by activated $T$ cell vaccines (excluding anti-id T cells) were, by definition, "anti-ergotypic" (20). Anti-erg T cells could then be defined functionally as T cells that respond by proliferation (with or without attendant cytokine production) to autologous or syngeneic activated $\mathrm{T}$ cells but not to the same $\mathrm{T}$ cells in a resting state. Circumstantial evidence indicated that anti-erg $T$ cells are widespread; human $T$ cells were also observed to proliferate in response to autologous $\mathrm{T}$ cells, provided the stimulators were activated (21). Anti-erg responses were found in persons irrespective of TCV or of autoimmune disease (22). Thus, anti-erg responses were not limited to experimental systems. The remainder of this review will discuss anti-erg $\mathrm{T}$ cells and their activities in immune regulation.

\section{Anti-erg ontogeny and T subset markers}

Anti-erg $\mathrm{T}$ cells can be detected in the thymuses, spleens, and lymph nodes of naive rats, independent of antigen priming. Indeed, thymocytes from 1-day-old rats manifest significant anti-ergotypic proliferative responses to activated, syngeneic T cells (manuscript submitted for publication). This illustrates the sensitivity of the anti-erg population; the only other $\mathrm{T}$ cell proliferative response that does not require pre-priming is the $\mathrm{T}$ cell response to foreign cells bearing major histocompatibility transplantation antigens. $\mathrm{T}$ cell proliferation in vitro to non-MHC molecules usually requires priming with the target antigen. Thus, the anti-erg response seems to equal the power of allo-MHC stimulation in vitro.

Naive anti-erg T cells were found to bear the $\mathrm{CD} 8^{+}$phenotype and included both TCR $\alpha / \beta^{+}$and TCR $\gamma / \delta^{+}$T cells (manuscript submitted for publication). The TCR $\gamma / \delta^{+}$anti-erg T cells secreted IFN- $\gamma$ and TNF- $\alpha$ in response to activated T cells; in contrast, the TCR $\alpha / \beta^{+}$ $\mathrm{T}$ cells proliferated but did not secret detectable cytokines. Thus, the anti-erg $\mathrm{T}$ cells of rats are much like anti-erg Tregs detected in humans (22). The interaction between naive anti-erg $\mathrm{T}$ cells and activated stimulator $\mathrm{T}$ cells can be achieved by direct $\mathrm{T}$ cell-T cell contact. Other APCs, such as macrophages, can present ergotope peptides to anti-erg $\mathrm{T}$ cells, but the response of anti-erg $\mathrm{T}$ cells to $\mathrm{APC} /$ peptide stimulation is not the same as their response to whole, activated T cells; this turns out to be important (see below). The response of naive TCR $\alpha / \beta^{+} \mathrm{CD}^{+}$anti-ergotypic T cells is restricted by classical MHC class I and dependent on B7 and CD28 molecules. The response of naive TCR $\gamma / \delta^{+}$anti-ergotypic T cells also depends on B7 and CD28 molecules but is not inhibited by antibodies to classical MHC class I or MHC class II molecules (manuscript submitted for publication); it remains to be seen whether such anti-erg $\mathrm{T}$ cells are restricted by nonclassical MHC molecules such as Qa-1/HLA-E. Note that the anti-ergotypic response is independent of the $\mathrm{CD} 4^{+} \mathrm{CD} 25^{+}$ population; the removal of $\mathrm{CD} 4^{+} \mathrm{CD} 25^{+} \mathrm{T}$ cells from naive populations did not affect the proliferation of anti-erg $\mathrm{T}$ cells in response to activated T cells (manuscript submitted for publication).

Following priming in vivo to an ergotope, the CD25 molecule (see below), anti-erg T cells also appear in the $\mathrm{CD}^{+}$subset of T cells and are restricted to classical MHC class II molecules (23). Thus, the anti-erg population is dynamic, and the anti-erg response seems to have a memory that includes the activation of $\mathrm{CD} 4^{+} \mathrm{T}$ cells. We shall now consider some of the different ergotopes recognized by anti-erg regulators.

\section{CD25 is an ergotope}

Naive anti-erg T cells, as mentioned above, do not reside in the $\mathrm{CD} 4^{+} \mathrm{CD} 25^{+}$population (manuscript submitted for publication), but the CD25 molecule itself is an ergotope (23). IL-2 is a growth factor for activated $\mathrm{T}$ cells, so it is not surprising that the IL-2 receptor (IL-2R) expressed by activated T cells harbors ergotopes. The expression of the IL-2R $\alpha$ (CD25) and IL-2R $\beta$ (CD122) chains by rat effector $\mathrm{T}$ cells is induced by activation, in contrast to the constitutive expression of the IL-2R $\gamma$ (CD132) chain (24); thus CD25 and CD122 might well function as ergotopes expressed by activated $T$ cells. Indeed, $T$ cell lines raised to immunogenic peptides of CD25 or CD122 were also found to respond to activated syngeneic $T$ cells, and a line raised to CD25 protected Lewis rats from EAE (25). These findings were extended by testing the ability of anti-CD25 anti-erg T cells to protect rats from adjuvant arthritis; it was found that the natural anti-erg response of naive rats spontaneously decreases upon the induction of adjuvant arthritis (manuscript submitted for publication, ref. 23). As discussed below, effector $\mathrm{T}$ cells can anergize anti-erg regulators; thus, a strong adjuvant arthritis effector response can overcome naive anti-erg $\mathrm{T}$ cell regulators. However, it was possible to strengthen the anti-erg $\mathrm{T}$ regulators by vaccinating rats actively with the $C D 25$ gene (23). This CD25 vaccination primed and enhanced the antierg $T$ cell proliferative response while it inhibited the induction of adjuvant arthritis. Moreover, when stimulated, both the anti-erg $\mathrm{T}$ cells and the otherwise arthritogenic effector $\mathrm{T}$ cells from the CD25-vaccinated rats secreted mainly IL-10, rather than IFN- $\gamma$ and TNF- $\alpha$. These results show that vaccination with a single ergotope, $\mathrm{CD} 25$, may induce protection from an experimental autoimmune disease by inducing a Th2-like cytokine shift in both the anti-erg response and the response to the antigens targeted in the disease (23). But effector $\mathrm{T}$ cells are not the only $\mathrm{T}$ cells that express the CD25 molecule; an important class of Tregs is the CD $4^{+} \mathrm{CD} 25^{+}$ population (26). If some anti-erg $\mathrm{T}$ cells recognize CD25, might they not affect these of regulators? Below, we shall discuss regulation of regulators.

\section{HSP60 is an ergotope}

$\mathrm{CD} 25$ is a reliable marker for activated $\mathrm{T}$ cells, but $\mathrm{T}$ cell ergotopes also include molecules that are not exclusive to $\mathrm{T}$ cells; heat shock protein 60 (HSP60) is a notable example. Because of the universal function of HSP60 as a molecular chaperone, variants of the HSP60 family are expressed by all cells, eukaryotic and prokaryotic (27). HSP60, however, functions not only as an intracellular chaperone, but also as a dominant signal molecule for the immune system. HSP60, self or foreign, is recognized by clones of T cells and B cells in the healthy immune repertoire (28). Moreover, HSP60 molecules, self and foreign, have been shown to function as ligands for activation of innate, toll-like receptor signaling in macrophages (29), DCs (30), and T cells (31). Additionally, vaccination with the HSP60 molecule (and some of its peptide fragments) can downregulate the autoimmune disease processes in rat adjuvant arthritis (32) and in type 1 diabetes in mice (33) and humans (34). Most relevant to the present discussion is our discovery that HSP60 epitopes can be presented by activated $\mathrm{T}$ cells as ergotopes to anti-erg regulators (manuscript in preparation). This work has not yet been published, 


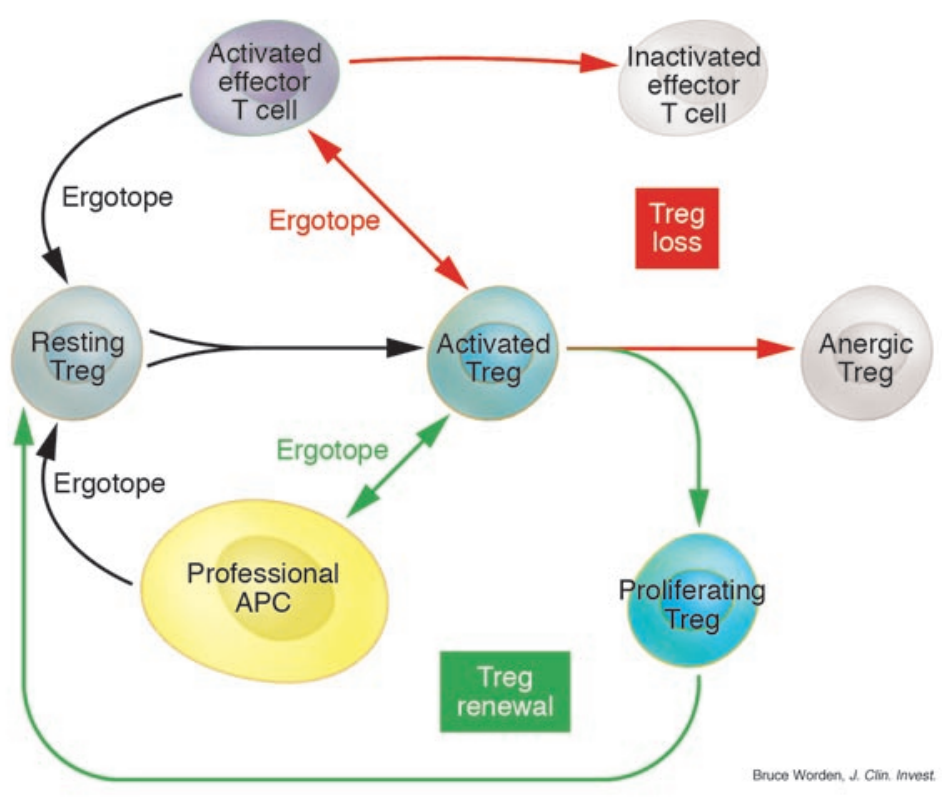

\section{Figure 1}

The balance between Treg loss (red arrows) and Treg renewal (green arrows). Resting Tregs can be activated by recognizing a specific ergotope presented in either of 2 ways: by an activated effector T cell or by a professional APC (yellow cell). The interaction between the activated Treg (green cells) and the activated effector T cell (purple cells) leads to inactivation of the effector T cell but also to anergy of the Treg (white cells). Thus, presentation of ergotopes by activated T cells leads to Treg loss. In contrast, an ergotope presented by a professional APC activates Treg proliferation and cycling (Treg renewal).

anti-erg T cells. Ergotopes are diverse molecules and include TCR, CD25, and HSP60 epitopes. The TCR molecule is expressed on resting $\mathrm{T}$ cells, but ergotypic TCR peptides are presented to anti-erg $\mathrm{T}$ cells only by activated $\mathrm{T}$ cells. This suggests that $\mathrm{T}$ cell activation affects TCR processing and/or TCR presentation. CD25 expression is itself linked to T cell activation. Unlike the TCR and CD25 molecules, HSP60 is not characteristic of T cells, but presentation of HSP60 peptides is a characteristic of activated and not of resting $T$ cells. Thus, there are many kinds of but the main findings can be summarized briefly. Lines of rat $\mathrm{T}$ cells specific for defined peptide epitopes of self-HSP60 proliferate in response to the particular HSP60 peptide presented by professional APCs, restricted by MHC class II. Yet these same T cell lines also respond to other activated, syngeneic $\mathrm{T}$ cell lines in the absence of any other APCs. This anti-erg response of the anti-HSP60 lines is also MHC class II restricted (activated rat T cells express MHC class II), but no peptide need be added to the reacting T cells; activated $T$ cells upregulate their expression of endogenous HSP60 and can present particular HSP60 peptides to the anti-HSP60, anti-erg T cell lines. The anti-HSP60 T cell lines do not respond to resting $T$ cells; only activated $T$ cells or T cell clones can present their HSP60 peptide epitopes to anti-erg T cells. Finally, the anti-HSP60, anti-erg $T$ cell lines can adoptively transfer resistance to the active induction of adjuvant arthritis in recipient rats.

\section{Ergotope diversity}

In summary, any molecule can serve as an ergotope if it fulfills 2 conditions: it is expressed and presented by activated $\mathrm{T}$ cells (and not by resting $\mathrm{T}$ cells), and it activates

\section{Figure 2}

Autoimmune disease through Treg loss and health through Treg renewal. Environmental factors (viral infection, toxic chemicals, trauma, etc.) can lead to triggering of an excess number of autoimmune effector T cells. These induce Treg anergy (see Figure 1) and Treg loss. The autoimmune effector excess can then bring about an autoimmune disease. In contrast, T cell and/or ergotope vaccination can activate Treg renewal (see Figure 1). The enhanced numbers of activated Tregs can inactivate autoimmune effector $T$ cells, leading to the restoration of health.

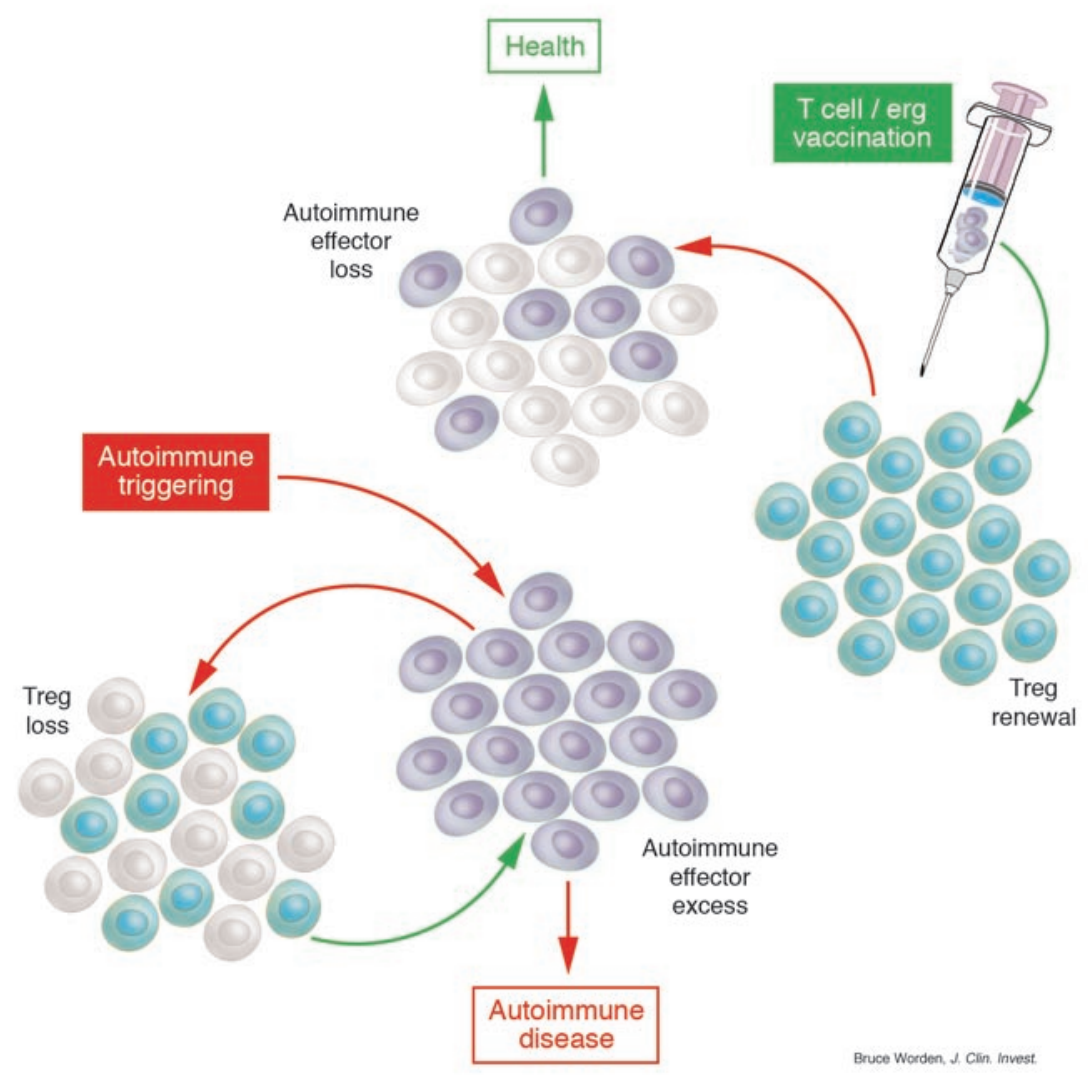




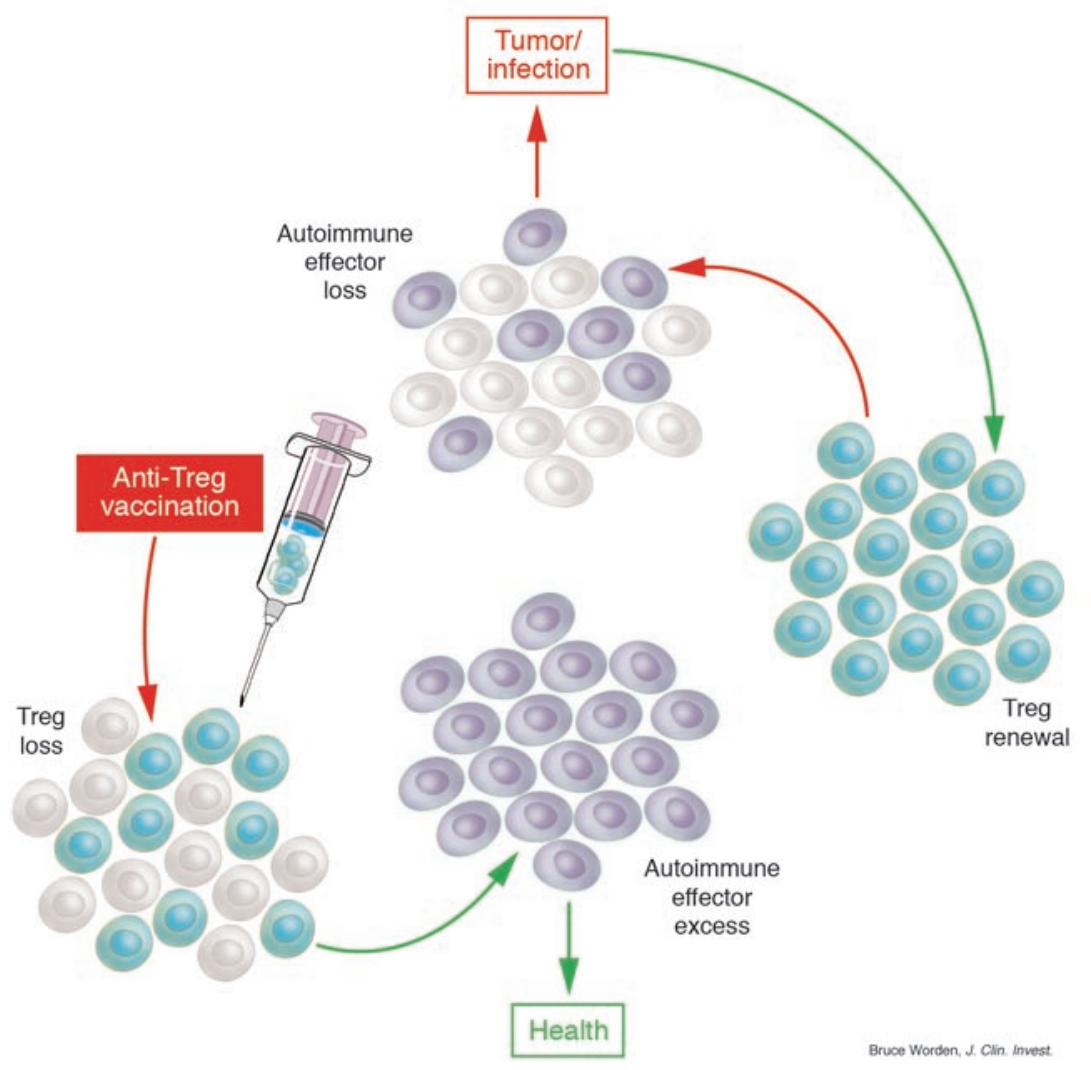

\section{Figure 3}

Immune evasion by tumors or infectious agents could take place by excess Treg renewal, resulting in suppression of specific effector T cells. AntiTreg vaccination could restore health by inducing Treg loss, leading to effector $T$ cell renewal and rejection of the tumor or infectious agent.

cytokines (IL-10 and TGF- $\beta$ ) when they meet activated effector $\mathrm{T}$ cells in an inflammatory site; the local anti-inflammatory cytokines modify effector $\mathrm{T}$ cell cytokine secretion, and the inflammation is suppressed.

\section{Regulating the regulators}

Above, we stated that anti-erg $\mathrm{T}$ cells can respond directly to ergotopes presented by whole, activated $\mathrm{T}$ cells and indirectly to APCs presenting ergotope peptides, but that the responses to these 2 forms of specific activation differ. The difference is critical in the life history of the anti-erg regulator. Anti-erg $\mathrm{T}$ cells that are activated by whole $\mathrm{T}$ cells do secrete their cytokines (IL-10 or TGF- $\beta$ ) and do downregulate their activated target $\mathrm{T}$ cells. But in the course of so effecting regulation, the anti-erg $\mathrm{T}$ cells themselves become anergic; they are extinguished by the effector cells they downregulate. Anti-erg T cells cannot be reactivated by a second contact with activated

Anti-erg T cells appear within the first day of life in the thymus, and so it is reasonable to suppose they are positively selected there. Thus, ergotopes are prominent among self-molecules for which there is positive autoimmunity, naturally (without autoimmune disease); ergotopes are members of the immunological homunculus (35).

\section{Mechanism of regulation}

It is not clear how anti-erg $\mathrm{T}$ cells actually regulate effector $\mathrm{T}$ cells. In fact, we know much more about immune effector machinery (cytolysis, cytokines, antibodies, etc.) than we do about any type of immune regulation. Anti-erg T cells are no exception. However, we can report 4 findings relevant to regulation. First, anti-erg Tregs, raised as lines (see below) or isolated from rats, can adoptively transfer resistance to adjuvant arthritis in naive rats (23). This means that the anti-erg $\mathrm{T}$ cells themselves can at least initiate the regulatory process. Second, natural anti-erg $\mathrm{T}$ cells can be boosted by specific stimulation with isolated ergotypic molecules - CD25, HSP60, or TCR epitopes (ref. 23 and manuscript in preparation). This means that anti-erg regulators respond immunologically to their targets. Third, activated anti-erg $\mathrm{T}$ cells secrete relatively large amounts of IL-10 (23) or TGF- $\beta$ (manuscript in preparation) when they respond to activated $T$ cells in vitro. This suggests that anti-erg $\mathrm{T}$ cells could regulate inflammatory reactions by modulating the local cytokine environment. Fourth, the autoimmune effector $\mathrm{T}$ cells from animals treated by anti-erg activation switch their response to their target antigens from a Th1-like response to a Th2-like response (23). This finding is compatible with the preceding point; anti-erg regulation affects the cytokine profile of the $\mathrm{T}$ cell response. Thus, we can imagine that anti-erg $\mathrm{T}$ cells can downregulate $\mathrm{T}$ cell inflammation by secreting anti-inflammatory effector $\mathrm{T}$ cells. In fact, any attempt to reactivate the anti-erg $\mathrm{T}$ cell regulators with whole $\mathrm{T}$ cells leads inevitably to the death of the anti-erg T cells. Over the years, this behavior effectively prevented researchers from raising lines of anti-erg $\mathrm{T}$ cells in vitro. Downregulation of anti-erg $\mathrm{T}$ cells by activated effector $\mathrm{T}$ cells might also explain how the induction of a disease such as adjuvant arthritis might downregulate naive anti-erg $\mathrm{T}$ cells (manuscript submitted for publication, ref. 23).

We have recently discovered that "cross-presentation" of ergotope peptides by APCs (rather than by activated T cells) stimulates the anti-erg $\mathrm{T}$ cells without causing them to become anergic and die (manuscript in preparation). Anti-erg activation by professional (non-T cell) APCs allows the cyclical self-renewal of the anti-erg T cells (see Figure 1). This observation has important implications.

First, the biology of the anti-erg response depends on the contextual signals within which the ergotope peptide-MHC signal is perceived. Effector T cells, in the course of their regulation, would seem to express signal molecules that affect the anti-erg regulators. We don't yet know what these accessory molecules are, but it has been observed in the past that direct $\mathrm{T}$ cell- $\mathrm{T}$ cell interactions lead to $\mathrm{T}$ cell inactivation (36). T cells can turn each other off. Downregulating the effectors can, in turn, downregulate their regulators, leading to Treg loss (Figure 1). This is reasonable; regulators have to be turned off so that future responses by effector cells will be possible. Unregulated regulators are as potentially dangerous as are unregulated effectors (see below). Once the activated effectors have been inactivated (or forced to undergo apoptosis), their ergotopes may be taken up and presented by scavenger APC types to allow the renewal and persistence of a small but requisite number of regulators. These renewed and quiescent regulators are 
now ready to be turned on (and then off) by future effector activation: Treg self-renewal (Figure 1).

Second, if the effectors can indeed regulate the numbers and the state of the regulators, then the distinction between regulator and effector might be more semantic than biologic. Regulators and effectors might not be fixed entities. Whether a $\mathrm{T}$ cell is a regulator or an effector would seem to depend on what you wish to measure and how you measure it. Our minds like to rest on neat dichotomies, but the regulator/effector distinction may have to go the way of other logically secure but biologically leaky categories (such as self/non-self, danger/non-danger, specific/degenerate) (37).

Third, the differential regulation of the regulators by whole $\mathrm{T}$ cells or by ergotope/APC stimulation may provide a way for us to modulate regulation therapeutically. We need to amplify regulation to shut down an autoimmune disease (Figure 2), but we need to arrest "aberrant" regulation if it allows a tumor to evade immune rejection or an infectious agent to reside in a sheltered niche (Figure 3).

\section{Upregulating regulation}

To increase the numbers of anti-erg (and anti-id) regulators in an autoimmune disease, we might consider adding ergotope peptide vaccines to $T$ cell vaccination (10) or to target-antigen vaccination (34). Ergotope peptides, delivered to the immune system via professional APCs, might amplify (by cyclical renewal) a deficient population of anti-erg regulators; Treg renewal could then shut off the autoimmune effector $\mathrm{T}$ cells and restore health (Figure 2).

\section{Downregulating regulation}

We shall close with a description of an experiment published a decade ago (38). Mycobacterium marinum multiplies logarithmically in BALB/c mice for about a month before immunity sets in and rejects the bacteria. We reasoned that, despite expression of powerful antigens such as bacterial HSP60 (30), the bacterium might temporarily evade the host immune response by activating host regulator/ suppressor T cells. Help the mouse get rid of its overly active regulation, and the mouse's immune system will get rid of the bacteria. So instead of vaccinating mice with bacterial antigens, we vaccinated prospective host mice with activated "suppressor" $\mathrm{T}$ cells obtained from the lymph nodes of syngeneic mice at the stage when they were harboring multiplying bacteria with no sign of immunity. The antireg vaccination apparently got rid of the excess suppression; when challenged, the vaccinated mice promptly rejected the bacteria: there was no evasion in these mice. T cells taken from immune mice, at the stage when the mice were not "suppressed," did not vaccinate naive recipient mice against bacterial challenge. Thus, it may be possible to abort a chronic microbial infection by immunizing the host against host Tregs exploited by the infectious agent to suppress the immune response; anti-Treg vaccination can induce Treg loss and so unleash suppressed effector cells to restore health (Figure 3). Now that regulation is reinstated (39), we can, when necessary, think about downregulating the regulators (40).

\section{Acknowledgments}

Irun R. Cohen is Mauerberger Professor of Immunology. The studies described here were supported in part by grants from the Center for the Study of Emerging Diseases and from the European Community.

Address correspondence to: Irun R. Cohen, Department of Immunology, The Weizmann Institute of Science, Rehovot 76100, Israel. Phone: 972-8-934-2911; Fax: 972-8-934-4103; E-mail: Irun.cohen@weizmann.ac.il.
1. Ben-Nun, A., Wekerle, H., and Cohen, I.R. 1981. The rapid isolation of clonable antigen-specific $T$ lymphocyte lines capable of mediating autoimmune encephalomyelitis. Eur. J. Immunol. 11:195-199.

2. Ben-Nun, A., Wekerle, H., and Cohen, I.R. 1981. Vaccination against autoimmune encephalomyelitis with T-lymphocyte line cells reactive against myelin basic protein. Nature. 292:60-61.

3. Cohen, I.R., Holoshitz J., van Eden, W., and Frenkel, A. 1985. T lymphocyte clones illuminate pathogenesis and affect therapy of experimental arthritis. Arthritis Rheum. 28:841-845.

4. Ben-Yehuda, A., et al. 1996. Lymph node cell vaccination against the lupus syndrome of MRL/lpr/lpr mice. Lupus. 5:232-236.

5. Elias, D., Tikochinski, Y., Frankel, G., and Cohen, I.R. 1999. Regulation of NOD mouse autoimmune diabetes by $\mathrm{T}$ cells that recognize a TCR CDR3 peptide. Int. Immunol. 11:957-966.

6. Zhang, J. 2002. T-cell vaccination for autoimmune diseases: immunologic lessons and clinical experience in multiple sclerosis. Expert Rev. Vaccines. 1:285-292.

7. Van der Aa, A., et al. 2003. T cell vaccination in multiple sclerosis patients with autologous CSFderived activated T cells: results from a pilot study. Clin. Exp. Immunol. 131:155-168.

8. Correale, J., et al. 2000. T cell vaccination in secondary progressive multiple sclerosis. J. Neuroimmunol. 24:130-139.

9. Kumar, V., Sercarz, E., Zhang, Z., and Cohen, I.R. 2001. T-cell vaccination: from basics to the clinic. Trends Immunol. 22:539-540.

10. Cohen, I.R. 2001. T-cell vaccination for autoimmune disease: a panorama. Vaccine. 20:706-710.

11. Lider, O., Reshef, T., Beraud, E., Ben-Nun, A., and Cohen, I.R. 1988. Anti-idiotypic network induced by $\mathrm{T}$ cell vaccination against experimental autoimmune encephalomyelitis. Science. 239:181-183.

12. Jiang, H., and Chess, L. 2000. The specific regulation of immune responses by CD8+ T cells restricted by the MHC class Ib molecule, Qa-1. Annu. Rev. Immunol. 18:185-216.

13. Kumar, V., et al. 1997. Recombinant T cell receptor molecules can prevent and reverse experimental autoimmune encephalomyelitis: dose effects and involvement of both CD4 and CD8 T cells. J. Immunol. 159:5150-5156.

14. Hu, D., et al. 2004. Analysis of regulatory CD8 T cells in Qa-1 deficient mice. Nat. Immunol. 5:516-523.

15. Panoutsakopoulou, V., et al. 2004. Suppression of autoimmune disease after vaccination with autoreactive T cells that express Qa-1 peptide complexes. J. Clin. Invest. 113:1218-1224. doi:10.1172/ JCI200420772.

16. Vandenbark, A.A., Hashim, G., and Offner, H. 1989. Immunization with a synthetic T-cell receptor Vregion peptide protects against experimental autoimmune encephalomyelitis. Nature. 12:541-544.

17. Kumar, V., Tabibiazar, R., Geysen, H.M., and Sercarz, E. 1995. Immunodominant framework region 3 peptide from TCR V beta 8.2 chain controls murine experimental autoimmune encephalomyelitis. J. Immunol. 154:1941-1950.

18. Naparstek, Y., et al. 1983. T lymphocyte lines producing or vaccinating against autoimmune encephalomyelitis (EAE). Functional activation induces peanut agglutinin receptors and accumulation in the brain and thymus of line cells. Eur. J. Immunol. 13:418-423.

19. Vaday, G.G., et al. 2001. Combinatorial signals by inflammatory cytokines and chemokines mediate leukocyte interactions with extracellular matrix. J. Leukoc. Biol. 69:885-892.
20. Lohse, A.W., Mor, F., Karin, N., and Cohen, I.R. 1989. Control of experimental autoimmune encephalomyelitis by $\mathrm{T}$ cells responding to activated T cells. Science. 244:820-822.

21. Russo, C., et al. 1981. Stimulation of human T lymphocytes by PHA-activated autologous $\mathrm{T}$ lymphocytes: analysis of the role of Ia-like antigens with monoclonal antibodies. Immunogenetics. 12:267-274.

22. Correale, J., Rojany, M., and Weiner, L.P. 1997. Human CD $8+$ TCR- $\alpha \beta(+)$ and TCR- $\gamma \delta(+)$ cells modulate autologous autoreactive neuroantigenspecific CD4+ T-cells by different mechanisms. J. Neuroimmunol. 80:47-64.

23. Mimran, A., et al. 2004. DNA vaccination with CD25 protects rats from adjuvant arthritis and induces an antiergotypic response. J. Clin. Invest. 113:924-932. doi:10.1172/JCI200417772.

24. Waldmann, T.A. 1993. The IL-2/IL-2 receptor system: a target for rational immune intervention. Immunol. Today. 14:264-270.

25. Mor, F., Reizis, B., Cohen, I,R., and Steinman, L. 1996. IL-2 and TNF receptors as targets of regulatory $\mathrm{T}-\mathrm{T}$ interactions: isolation and characterization of cytokine receptor-reactive $T$ cell lines in the Lewis rat. J. Immunol. 157:4855-4861.

26. Sakaguchi, S. 2000. Regulatory T cells: key controllers of immunologic self-tolerance. Cell. 101:455-458.

27. Kaufman, B.A., Kolesar, J.E., Perlman, P.S., and Butow R.A. 2003. A function for the mitochondrial chaperonin Hsp60 in the structure and transmission of mitochondrial DNA nucleoids in Saccharomyces cerevisiae. J. Cell Biol. 163:457-461.

28. Amir-Kroll, H., Nussbaum, G., and Cohen, I.R. 2003. Proteins and their derived peptides as carriers in a conjugate vaccine for streptococcus pneu- 
moniae: self-heat shock protein 60 and tetanus toxoid. J. Immunol. 170:6165-6171.

29. Ohashi, K., Burkart, V., Flohe, S., and Kolb, H. 2000. Heat shock protein 60 is a putative endogenous ligand of the toll-like receptor- 4 complex. J. Immunol. 164:558-561.

30. Flohe, S.B., et al. 2003. Human heat shock protein 60 induces maturation of dendritic cells versus a Th1promoting phenotype. J. Immunol. 170:2340-2348.

31. Zanin-Zhorov, A., Nussbaum, G., Franitza, S., Cohen, I.R., and Lider, O. 2003. T cells respond to heat shock protein 60 via TLR2: activation of adhesion and inhibition of chemokine receptors. FASEBJ. 17:1567-1569.

32. Quintana, F.J., Carmi, P., Mor, F., and Cohen, I.R.
2003. DNA fragments of the human $60-\mathrm{kDa}$ heat shock protein (HSP60) vaccinate against adjuvant arthritis: identification of a regulatory HSP60 peptide. J. Immunol. 171:3533-3541.

33. Quintana, F.J, Carmi, P., and Cohen, I.R. 2002. DNA vaccination with heat shock protein 60 inhibits cyclophosphamide-accelerated diabetes. J. Immunol. 169:6030-6035.

34. Raz, I., et al. 2001. Beta-cell function in newonset type 1 diabetes and immunomodulation with a heat-shock protein peptide (DiaPep277): a randomised, double- blind, phase II trial. Lancet. 358:1749-1753.

35. Cohen, I.R. 1992. The cognitive paradigm and the immunological homunculus. Immunol. Today.
13:490-494.

36. Brod, S.A., Purvee, M., Benjamin, D., and Hafler, D.A. 1990. T-T cell interactions are mediated by adhesion molecules. Eur. J. Immunol. 20:2259-2268.

37. Cohen, I.R. 2000. Discrimination and dialogue in the immune system. Semin. Immunol. 12:215-279, 321-323.

38. Levy, L., Enk, C.D., Zipris, D., and Cohen, I.R. 1994. Protection of mice against mycobacterial infection by lymphoid cell vaccination. Isr. J. Med. Sci. 30:22-25.

39. Cantor, H. 2004. Reviving suppression? Nat. Immunol. 5:347-348.

40. Cohen, I.R. 2000. Tending Adam's Garden: Evolving the Cognitive Immune Self. Academic Press. London, United Kingdom. 266 pp. 